\title{
Anisotropic and inhomogeneous cosmologies
}

\author{
M.A.H. MACCALLUM \\ School of Mathematical Sciences \\ Queen Mary and Westfield College, University of London \\ Mile End Road, London E1 4NS, U.K. \\ E-mail: M.A.H.MacCallum@qmw.ac.uk
}

\section{INTRODUCTION}

My first impressions of Dennis Sciama came from a short introductory astrophysics course he gave to undergraduates in 1964. Then in 1966-7 I took his Cambridge Part III course in relativity, in which he charitably ignored my inadvertent use of Euclidean signature in the examination (an error I spotted just at the very end of the allowed time) and gave me a good mark. In both these courses he showed the qualities of enthusiasm and encouragement of students with which I was to become more familiar later in 1967 when I began as a research student. A project on stellar structure had taught me that I did not want to work on that, and I began under Dennis with the idea of looking at galaxy formation. However, by sharing an office with John Stewart I came to read John's paper with George Ellis Stewart and Ellis, 1968 and its antecedent Ellis, 1967 and developed an interest in relativistic cosmological models, which led to George becoming my second supervisor.

I was still in Sciama's group, and I learnt a lot from the tea-table conversations, which seemed to cover all of general relativity and astrophysics. Dennis taught us by example that the field should not be sub-divided into mathematics and physics, or cosmological and galactic and stellar, but that one needed to know about all those things to do really good work. Of course he was not uncritically enthusiastic: his own opinions were strongly enough held that we used to joke that if we wanted to stop research all we need do was say loudly in the tea-room that we did not believe in Mach's principle. But it was a very supportive and stimulating atmosphere for which I will always be grateful.

This is a review of what we have learnt from the study of non-standard cos- 
mologies in which I got involved 25 years ago. Only exact solutions will be considered: the perturbation theory will be left for others to discuss. In earlier reviews [MacCallum, 1979, MacCallum, 1984] I started from the mathematical classification of the solutions but here I want to take a different route and consider the application areas. So let me just quickly remind readers of the general groups of models to which I will later refer. They are:

[1] Spatially-homogeneous and isotropic models. In relativity these give the FriedmanLemaître-Robertson-Walker cosmologies, and the "standard model".

[2] Spatially-homogeneous but anisotropic models. These are the Bianchi models, in general, the exceptions being the Kantowski-Sachs models with an $S^{2} \times R^{2}$ topology.

[3] Isotropic but inhomogeneous models. These are spherically symmetric models, whose dust subcases, having been first discussed by Lemaître (1933), are called Tolman-Bondi models.

[4] Models with two ignorable coordinates, usually with a pair of commuting Killing vectors. These may be plane or cylindrically symmetric.

[5] Models with less symmetry than those above. Only a few special cases are known exactly.

In giving this review I only had time to mention and discuss some selected papers and issues, not survey the whole vast field. Thus the bibliography is at best a representative selection from many worthy and interesting papers, and authors whose work is unkindly omitted may quite reasonably feel it is unrepresentative.

\section{OBSERVATIONS, THE STANDARD MODEL AND ALTERNATIVES}

What is it that a cosmological model should explain? There are the following main features:

[1] Lumpiness, or the clumping of matter. The evidence for this is obvious.

[2] Expansion, shown by the Hubble law.

[3] Evolution, shown by the radio source counts and more recently by galaxy counts.

[4] A hot dense phase, to account for the cosmic microwave background radiation (CMWBR) and the abundances of the chemical elements.

[5] Isotropy, shown to a high degree of approximation in various cosmological observations, but especially in the CMWBR.

[6] Possibly, homogeneity. (The doubt indicated here will be explained later.) 
[7] The numerical values of parameters of the universe and its laws, such as the baryon number density, the total density parameter $\Omega$, the entropy per baryon, and the coupling constants

[8] (Perhaps) such features as the presence of life.

The standard big-bang model at the time I started as a student was:

[1] Isotropic at all points and thus necessarily...

[2] Spatially-homogeneous, implying Robertson-Walker geometry.

[3] Satisfied Einstein's field equations

[4] At recent times (for about the last $10^{10}$ years) pressureless and thus governed by the Friedman-Lemaître dynamics.

[5] At early times, radiation-dominated, giving the Tolman dynamics and a thermal history including the usual account of nucleogenesis and the microwave background.

To this picture, which was the orthodox view from about 1965-80, the last decade added the following extra orthodoxies:

[6] $\Omega=1$. Thus there is dark matter, for which the Cold Dark Matter model was preferred.

[7] Inflation - a period in the early universe where some field effectively mimics a large cosmological constant and so causes a period of rapid expansion long enough to multiply the initial length scale many times.

[8] Non-linear clustering on galaxy cluster scales, modelled by the $N$-body simulations which fit correlation functions based on observations.

and also added, as alternatives, such concepts as cosmic strings, GUTs or TOEST and so on.

The standard model has some clear successes: it certainly fits the Hubble law, the source count evolutions (in principle if not in detail), the cosmic microwave spectrum, the chemical abundances, the measured isotropies, and the assumption of homogeneity. Perhaps its greatest success was the prediction that the number of neutrino species should be 3 and could not be more than 4 , a prediction now fully borne out by the LEP data.

However, the model still has weaknesses [MacCallum, 1987]. For example, the true clumping of matter on large scales, as shown by the QDOT data [Saunders et al., 1991] and the angular correlation functions of galaxies [Maddox et al., 1990], is too strong

\footnotetext{
${ }^{1}$ Why so anatomical?
} 
for the standard cold dark matter account?2]. The uniformity of the Hubble flow is under question from the work of the "Seven Samurai" [Lynden-Bell et al., 1988] and others. The question of the true value of $\Omega$ has been re-opened, partly because theory has shown that inflation does not uniquely predict $\Omega=1$ and partly because observations give somewhat variant values. Some authors have pointed out that our knowledge of the physics valid at nucleogenesis and before is still somewhat uncertain, and we should retain some agnosticism towards our account of those early times.

Finally, we should recognize that our belief in homogeneity has very poor observational support. We have data from our past light cone (and those of earlier human astronomers) and from geological records Hoyle, 1962. Studying homogeneity requires us to know about conditions at great distances at the present time, whereas what we can observe at great distances is what happened a long time ago, so to test homogeneity we have to understand the evolution both of the universe's geometry

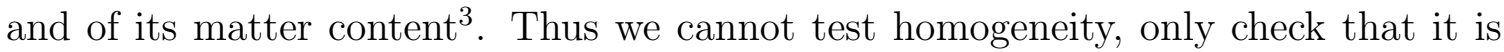
consistent with the data and our understanding of the theory. The general belief in homogeneity is indeed like the zeal of the convert, since until the 1950s, when Baade revised the distance scale, the accepted distances and sizes of galaxies were not consistent with homogeneity.

These comments, however, are not enough to justify examination of other models. Why do we do that? I think there are several reasons. Alternative models provide fully non-linear modelling of local processes. They may show whether characteristics thought to be peculiar to the standard model, and thus a test of it, can occur elsewhere. They may be used in attempted proofs that no universe could be anisotropic or inhomogeneous, by proving that any strong departures from the standard model decay away during evolution. They can be comparators in data analysis, to show that only standard models fit. Finally, they may even be advanced as replacements of the standard model.

Before starting to examine how the alternatives fare in these various rôles, I must point out two major defects in work up to now. One is that the matter content is almost always assumed to be a perfect fluid. Yet even in the simplest nonstandard models, the Bianchi models, as soon as matter is in motion relative to the homogeneous surfaces (i.e. becomes 'tilted') it experiences density gradients which

\footnotetext{
${ }^{2}$ These discoveries made it possible for disagreement with the 1980s dogmatism on such matters to at last be listened to.

${ }^{3}$ Local measures of homogeneity merely tell us that the spatial gradients of cosmic quantities are not too strong near us.

${ }^{4}$ Such models have recently been used to fit the observed dipole anisotropy in the CMWBR Turner, 1992, though other explanations seem to me more credible.
} 
should lead to heat fluxes Bradley and Sviestins, 1984]: similar remarks apply to other simple models. Attempting to remedy this with some other mathematically convenient equation of state is not an adequate response; one must try to base the description of matter on a realistic model of microscopic physics or thermodynamics, and few have considered such questions Salvati et al., 1987, Bona and Coll, 1988, Romano and Pavon, 1992. The other objection is that we can only explore the mathematically tractable subsets of models, which may be far from representative of all models.

\section{MODELLING LOCAL NON-LINEARITIES}

Cosmic strings have been modelled by cylindrically symmetric models, starting with the work of Gott, Hiscock and Linet in 1985. These studies have usually been done with static strings quantum fields in the neighbourhood of the string.

Similarly, exact solutions for domain walls, using plane symmetric models, usually static, have been considered [Vilenkin, 1983, "pser and Sikivie, 1984, Goetz, 1990, Wang, 1991] [0.

Galactic scale inhomogeneities have frequently been modelled by spherically symmetric models, usually Tolman-Bondi. They have been used to study galaxy formation (e.g. Tolman (1934), Carr and Yahil (1990), and Meszaros (1991)), to estimate departures from the simple theory of the magnitude-redshift relations based on a smoothed out model] (e.g. Dyer (1976), Kantowski (1969b) and Newman (1979): note that these works show that the corrections depend on the choice of modelling), and as the simplest models of gravitational lenses 8 . Spherically symmetric inhomogeneities have also been used to model the formation of primordial black holes Carr and Hawking, 1974.

On a larger scale still inhomogeneous spacetimes have been used to model clusters

\footnotetext{
${ }^{5}$ There is some controversy about whether these can correctly represent strings embedded in an expanding universe Clarke et al., 1990.

${ }^{6}$ Note that since the sources usually have a boost symmetry in the timelike surface giving the wall, corresponding solutions have timelike surfaces admitting the (2+1)-dimensional de Sitter group.

${ }^{7}$ The point is that the beams of light we observe are focussed only by the matter actually inside the beam, not the matter that would be there in a completely uniform model.

${ }^{8}$ The very detailed modern work interpreting real lenses to study various properties of individual sources and the cosmos mostly uses linearized approximations.
} 
of galaxies [Kantowski, 1969a], variations in the Hubble flow due to the supercluster [Mavrides, 1977], the evolution of cosmic voids [Sato, 1984, Hausman et al., 1983, Bonnor and Chamorro, 1990 and 1991, the observed distribution of galaxies and simple hierarchical models of the universe [Bonnor, 1972, Wesson, 1978, Wesson, 1979, Ribeiro, 1992a.

The references just cited are only the tip of the iceberg. For his mammoth survey of all inhomogeneous cosmological models which contain, as a limiting case, FriedmanRobertson-Walker models, Krasinski now has read about 1900 papers (as reported at the GR13 conference in 1992 [9]). As well as the issues mentioned above, these papers discuss many others including models for interactions between different forms of matter, generation of gravitational radiation, and the nature of cosmic singularities.

There is not enough space here to do all these arguments justice, and anyway it would be unfair to pre-empt Krasinski's conclusions. Moreover, I believe the issues for which I have given a few detailed references are (together with some appearing later in this survey) the most important astrophysically. So I will just mention two more points which have arisen. One is that some exact non-linear solutions obey exactly the linearized perturbation equations for the FLRW models Goode and Wainwright, 1982, Carmeli et al., 1983]. The other is a jeu d'esprit in which it was shown that in a "Swiss cheese" model, made by joining two FLRW exteriors at the two sides of a Kruskal diagram for the Schwarzschild solution, one can have two universes each of which can receive (but not answer) a signal from the other Sussman, 1985.

\section{WHICH FLRW PROPERTIES ARE SPECIAL?}

The earliest use of anisotropic cosmological models to study a real cosmological problem was the investigation by Lemaitre (1933) of the occurrence of singularities in Bianchi type I models. The objective was to explore whether the big-bang which arose in FLRW models was simply a consequence of the assumed symmetry: it was of course found not to be.

A later similar investigation was to see if the helium abundance, as known in the 1960s, could be fitted better by anisotropic cosmologies than by FLRW models, which at the time appeared to give discrepancies. The reason this might happen is

\footnotetext{
${ }^{9}$ The survey is not yet complete and remains to be published, but interim reports have appeared in some places, e.g. Krasinski, 1990.
} 
that anisotropy speeds up the evolution between the time when deuterium can first form, because it is no longer dissociated by the photons, and the time when neutrons and protons are sufficiently sparse that they no longer find each other to combine. Hawking and Tayler (1966) were pioneers in this effort, which continued into the 1980s but suffered some mutations in its intention.

First the argument was reversed, and the good agreement of FLRW predictions with data was used to limit the anisotropy during the nucleogenesis period (see e.g. Barrow (1976), Olson (1977)). Later still these limits were relaxed as a result of considering the effects of anisotropic neutrino distribution functions Rothman and Matzner, 1982 and other effects on reaction rates Juszkiewicz et al., 1983. It has even been shown [Matravers et al., 1984, Barrow, 1984 that strongly anisotropic models, not obeying the limits deduced from perturbed FLRW models, can also produce correct element abundances, though they may violate other constraints Matravers and Madsen, 1985, Matravers et al., 1985.

The above properties turn out not to be special to FLRW geometry. One that might be thought to be is the exact isotropy of the CMWBR. To test this, many people in the 1960s and 70s computed the angular distribution of the CMWBR temperature in Bianchi models (e.g. Thorne (1967), Novikov (1968), Collins and Hawking (1972), and Barrow et al. (1985)). These calculations allow limits to be put on small deviations from isotropy from observation, and also enabled, for example, the prediction of 'hot spots' in the CMWBR in certain Bianchi models, which could in principle be searched for, if there were a quadrupole component $\square$, to see if the quadrupole verifies one of those models.

Similar calculations, by fewer people, considered the polarization Rees, 1968, Anile, 1974, Tolman and Matzner, 1984 and spectrum Rees, 1968, Rasband, 1971. More recently still, work has been carried out on the microwave background in some inhomogeneous models Saez and Arnau, 1990. It has been shown that pure rotation (without shear) is not ruled out by the CMWBR Obukhov, 1992, but this result may be irrelevant to the real universe where shear is essential to non-trivial perturbations [Goode, 1983, Dunsby, 1992].

One property, the nature of the big-bang singularity, as distinct from its existence, has been so extensively discussed as to demand a section of its own.

\footnotetext{
${ }^{10}$ Which, since the meeting this survey was given at, has been shown to exist in the COBE data.
} 


\section{THE ASYMPTOTIC BEHAVIOUR OF CLAS- SICAL COSMOLOGIES}

One can argue that classical cosmologies are irrelevant before the Planck time, but until a theory of quantum gravity is established and experimentally verified (if indeed that will ever be possible) there will be room for discussions of the behaviour of classical models near their singularities.

In the late 1950s and early 60s Lifshitz and Khalatnikov and their collaborators showed (a) that singularities in synchronous coordinates in inhomogeneous cosmologies were in general 'fictitious' and (b) that a special subclass gave real curvature singularities Lifshitz and Khalatnikov, 1963. From these facts they (wrongly) inferred that general solutions did not have singularities. This contradicted the later singularity theorems (for which see Hawking and Ellis (1973)), a disagreement which led to the belief that there were errors in LK's arguments. They themselves, in collaboration with Belinskii, and independently Misner, showed that Bianchi IX models gave a more complicated, oscillatory, behaviour than had been discussed in the earlier work, and Misner christened this the 'Mixmaster' universe after a brand of food mixer.

The detailed behaviour of the Mixmaster model has been the subject of stillcontinuing investigations: some authors argue that the evolution shows ergodic and chaotic properties, while others have pointed out that the conclusions depend crucially on the choice of time variable [Barrow, 1982, Burd et al., 1990, Berger, 1991]. Numerical investigations are tricky because of the required dynamic range if one is to study an adequately large time-interval, and the difficulties of integrating chaotic systems.

The extension of these ideas to the inhomogeneous case, by Belinskii, Lifshitz and Khalatnikov, has been even more controversial, though prompting a smaller literature. It was strongly attacked by Barrow and Tipler (1979) on a number of technical grounds, but one can take the view that these were not as damaging to the case as Barrow and Tipler suggested [Belinskii et al., 1980, MacCallum, 1982]. Indeed the 'velocity-dominated' class whose singularities are like the Kasner (vacuum Bianchi) cosmology have been more rigorously characterized and the results justified [Eardley et al., 1971, Holmes et al., 1990]. Sadly this does not settle the more general question, and attempts to handle the whole argument on a completely rigorous footing $\square$ have so far failed.

\footnotetext{
${ }^{11}$ One of them made by Smallwood and myself.
} 
General results about singularity types have been proved. The 'locally extendible' singularities, in which the region around any geodesic encountering the singularity can be extended beyond the singular point, can only exist under strong restrictions [Clarke, 1976], while the 'whimper' singularities [King and Ellis, 1973], in which curvature invariants remain bounded while curvature components in some frames blow up, have been shown to be non-generic and unstable [Siklos, 1978. Examples of these special cases were found among Bianchi models, and both homogeneous and inhomogeneous cosmologies have been used as examples or counter-examples in the debate.

A further stimulus to the study of singularities was provided by Penrose's conjecture that gravitational entropy should be low at the start of the universe and this would correspond to a state of small or zero Weyl tensor [Penrose, 1979, Tod, 1992].

Studies of the behaviour of Bianchi models have been much advanced by the adoption of methods from the theory of dynamical systems. In the early 70 s this began with the discussion of phase portraits for special cases Collins, 1971] and was extended in work in which (a) the phase space was compactified, (b) Lyapunov functions, driving the system near the boundaries of the phase space, were found and (c) analyticity together with the behaviour of critical points and separatrices was used to derive the asymptotic behaviour [Bogoyavlenskii, 1985. In the last decade these methods have been coupled with the parametrization of the Bianchi models using automorphism group variables [Collins and Hawking, 1973, Harvey, 1979, Jantzen, 1979, Siklos, 1980, Roque and Ellis, 1985, Jaklitsch, 1987.

The automorphism group can be briefly described as follows. Writing the Bianchi metrics as

$$
d s^{2}=-d t^{2}+g_{\alpha \beta}(t)\left(e^{\alpha}{ }_{\mu} d x^{\mu}\right)\left(e^{\beta}{ }_{\nu} d x^{\nu}\right)
$$

where the corresponding basis vectors $\left\{\mathbf{e}_{\alpha}\right\}$ obey

$$
\left[\mathbf{e}_{\alpha}, \mathbf{e}_{\beta}\right]=C_{\alpha \beta}^{\gamma} \mathbf{e}_{\gamma}
$$

in which the C's are the structure constants of the relevant symmetry group, one uses a transformation

$$
\hat{\mathbf{e}}^{\alpha}=M_{\beta}^{\alpha} \mathbf{e}^{\beta}
$$

chosen so that the $\left\{\hat{\mathbf{e}}_{\alpha}\right\}$ obey the same commutation relations as the $\left\{\mathbf{e}_{\alpha}\right\}$. The matrices $M$ are time-dependent and can be chosen so that the new metric coefficients $\hat{g}_{\alpha \beta}$ take some convenient form. The real dynamics is in these metric coefficients. The idea is present in earlier treatments which grew from Misner's methods for the Mixmaster case [Ryan and Shepley, 1975] but unfortunately the type IX case was 
highly misleading in that for Bianchi IX (and no others except Bianchi I) the rotation group is an automorphism group.

A long series of papers by Jantzen, Rosquist and collaborators DJantzen, 1984, Rosquist et al., 1990 have coupled these ideas with Hamiltonian treatments in a powerful formalism. Using a different, and in some respects simpler, set of variables, Wainwright has also attacked the asymptotics problem Wainwright and Hsu, 1989: his variables are well-suited for those questions because their limiting cases are physical evolutions of simpler models rather than singular behaviours.

The conclusions of these studies have justified the work of Belinskii et al. for the homogeneous case (but do not affect the arguments about the inhomogeneous cases) and have enabled new exact solutions to be found and some general statements about the occurrence of these solutions (which in general have self-similarity in time) to be made Wainwright and Hsu, 1989, in particular showing their rôles as attractors of the dynamical systems.

The other class of models where techniques have improved considerably are the models with two commuting Killing vectors, even when these vectors are not hypersurfaceorthogonal. Some studies have focussed on the mathematics, showing how known vacuum solutions can be related by solution-generating techniques [Kitchingham, 1984], while others have concentrated on the physics of the evolution of fluid models (not obtainable by generating techniques, except in the case of 'stiff' fluid, $p=\rho$ ) and interpretative issues [Wainwright and Anderson, 1984, Hewitt et al., 1991]. It emerges that the models studied are typically Kasner-like near the singularity (agreeing with the LK arguments), and settle down to self-similar or spatially homogeneous models with superposed high-frequency gravitational waves at late times. However, some cases have asymptotic behaviour near the singularity like plane waves, and others are non-singular Chinea et al., 1991. The Penrose conjecture has been particularly developed, using exact solutions as examples, by Wainwright and Goode, who have given a precise definition to the notion of an 'isotropic singularity' Goode et al., 1992, Tod, 1992.

Many authors have also considered the far future evolution (or, in closed models, the question of recollapse, whose necessity in Bianchi IX models lacked a rigorous proof until recently [Lin and Wald, 1991]). From various works [MacCallum, 1971, Collins and Hawking, 1973, Barrow and Tipler, 1978 one finds that the homogeneous but anisotropic models do not in general settle down to an FLRW-like behaviour but typically generate shears of the order of $25 \%$ of their expansion rates. 
This last touches on an interesting question about our account of the evolution of the universe: is it structurally stable, or would small changes in the theory of the model parameters change the behaviour grossly? Several instances of the latter phenomenon, 'fragility', have recently been explored by Tavakol, in collaboration with Coley, Ellis, Farina, Van den Bergh and others Coley and Tavakol, 1992].

\section{DO NON-FLRW MODELS BECOME SMOOTH?}

Attempts to smoothe out anisotropies or inhomogeneities by any process obeying deterministic sets of differential equations satisfying Lipschitz-type conditions are doomed to fail, as was first pointed out by Collins and Stewart (1971) in the context of viscous mechanisms. The argument is simply that one can impose any desired amount of anisotropy or inhomogeneity now and evolve the system backwards in time to reach initial conditions at some earlier time whose evolution produces the chosen present-day values. This was one of the arguments which rebutted Misner's ingenious suggestion that viscosity in the early universe could explain the present level of isotropy and homogeneity regardless of the initial conditions.

The same argument also holds for inflationary models. Inflation in itself, without the use of singular equations or otherwise indeterminate evolutions, cannot wholly explain present isotropy or homogeneity, although it may reduce deviations by large factors Sirousse-Zia, 1982, Wald, 1983, Moss and Sahni, 1986, Futamase et al., 1989. Objections to some specific calculations have been given Rothman and Ellis, 1986. Although one can argue that anisotropy tends to prolong inflation, this does not remove the difficulty.

Since 1981 I have been arguing a heretical view about one of the grounds for inflation, namely the 'flatness problem', on the grounds that the formulation of this problem makes an implicit and unjustified assumption that the a priori probabilities of values of $\Omega$ is spread over some range sufficient to make the observed closeness to 1 implausible. Unless one can justify the a priori distribution, there is no implausibility[²] Ellis, 1991.

However, if one accepts there is a flatness problem, then there is also an isotropy

\footnotetext{
${ }^{12}$ One can however argue that only $\Omega=1$ is plausible, on the grounds that otherwise the quantum theory before the Planck time would have to fix a length-scale parameter much larger than any quantum scale, only the $\Omega=1$ case being scale-free. I am indebted to Gary Gibbons for this remark.
} 
problem, since at least for some probability distributions on the inhomogeneity and anisotropy the models would not match observation. Protagonists of inflation cannot have it both ways. Perhaps, if one does not want to just say "well, that's how the universe was born", one has to explain the observed smoothness by appeal to the 'speculative era', as Salam (1990) called it, i.e. by appeal to one's favourite theory of quantum gravity.

Incidentally, one may note that inflation does not solve the original form of the 'horizon problem', which was to account completely for the similarity of points on the last scattering surface governed by different subsets of the inital data surface. Inflation leads to a large overlap between these initial data subsets, but not to their exact coincidence. Thus one still has to assume that the non-overlap regions are not too different. While this may give a more plausible model, it does not remove the need for assumptions on the initial data.

A further interesting application of non-standard models has come in a recent attempt to answer the question posed by Ellis and Rothman (unpublished) of how the universe can choose a uniform reference frame at the exit from inflation when a truly de Sitter model has no preferred time axis. Anninos et al. (1991a) have shown by taking an inflating Bianchi $\mathrm{V}$ model that the answer is that the memory is retained and the universe is never really de Sitter.

Finally, one may comment that if inflation works well at early times, then inflation actually enhances the chance of an anisotropic model fitting the data, and that since the property of anisotropy cannot be totally destroyed in general (because it is coded into geometric invariants which cannot become zero by any classical evolution) the anisotropy could reassert itself in the future!

\section{ASTROPHYSICAL AND OBSERVABLE CONSEQUENCES OF NON-STANDARD MODELS}

Galaxy formation in anisotropic models has been studied to see if they could overcome the well-known difficulties of FLRW models (without inflation), but with negative results Perko et al., 1972].

As mentioned above solutions with two commuting Killing vectors provide models 
for universes with gravitational waves. Aspects of these models have been considered by several authors, e.g. Carr and Verdaguer (1983), Ibanez and Verdaguer (1983), Feinstein (1988). There are in fact several mathematically related but physically distinct classes of solutions of the Einstein equations accessible by generating techniques: stationary axisymmetric spacetimes, colliding wave solutions (nicely summarized in Griffiths (1991) and Ferrari (1990)), and cosmological solutions, the differences arising from the timelike or spacelike nature of the surfaces of symmetry and the nature of the gradient of the determinant of the metric in those surfaces.

The generating techniques essentially work for forms of matter with characteristic propagation speed equal to the velocity of light, and use one or more of a battery of related techniques: Bäcklund transformation, inverse scattering, soliton solutions and so on. One interesting question that has arisen from recent work is whether solitons in relativity do or do not exhibit non-linear interactions: Boyd et al. (1991), in investigations of solitons in a Bianchi I background, found no non-linearity, while Belinskii (1991) has claimed there is a non-linear effect.

Work on the observable consequences of non-standard models has been done by many, as mentioned above. One intriguing possibility raised by Ellis et al. (1978) is that the observed sphere on the last scattering surface could lie on a timelike (hyper)cylinder of homogeneity in a static spherically symmetric model. This makes the CMWBR isotropic at all points not only at the centre, and although it cannot fit all the other data, the model shows how careful one must be, in drawing conclusions about the geometry of the universe from observations, not to assume the result one wishes to prove.

Recent work by Ribeiro (1992b), in the course of an attempt to make simple models of fractal cosmologies using Tolman-Bondi metrics, has reminded us of the need to compare data with relativistic models not Newtonian approximations. Taking the Einstein-de Sitter model, and integrating down the geodesics, he plotted the number counts against luminosity distances. At small distances, where a simple interpretation would say the result looks like a uniform density, the graph is irrelevant because the distances are inside the region where the QDOT survey shows things are lumpy Saunders et al., 1991, while at greater redshifts the universe ceases to have a simple power-law relation of density and distance. Thus even Einstein-de Sitter does not look homogeneous!

One must therefore ask in general "do homogeneous models look homogeneous?". Of course, they will if the data is handled with appropriate relativistic corrections, but to achieve such comparisons in general requires the integration of the null geodesic 
equations in each cosmological model considered, and, as those who have tried it know, even when solving the field equations is simple, solving the geodesic equations may not be.

Ultimately we will have to refine our understanding with the help of numerical simulations which can include fully three-dimensional variations in the initial data, and some excellent pioneering work has of course been done, e.g. Anninos et al. (1991b), but capabilities are still limited (for example Matzner (1991) could only use a space grid of $31^{3}$ points and 256 time steps). Moreover before one can rely on numerical simulations one needs to prove some structural stability results.

\section{IS THE STANDARD MODEL RIGHT?}

While I do not think one can give a definitive answer to this question, I would personally be very surprised if anisotropic but homogeneous models turned out to be anything more than useful examples. However, the status of fully inhomogeneous models is less clear.

One argument is that while the standard models may be good approximations at present, they are unstable to perturbations both in the past and the future. The possible alternative pasts are quite varied, as shown in section 5 , even without considering quantum gravity. Similarly, as also mentioned in section 5, the universe may not be isotropic in the far future. Moreover, there is the question of on what scale, if any, the FLRW model is valid. Its use implies some averaging, and is certainly not correct on small scales. Is it true on any scale? If so, on what scales? There may be an upper as well as a lower bound, since we have no knowledge of conditions outside our past null cone, where some inflationary scenarios would predict bubbles of differing FLRW universes, and perhaps domain walls and so on.

If the universe were FLRW, or very close to that, this means it is in a region, in the space of all possible models, which almost any reasonable measure is likely to say has very low probability (though note the remarks on assignments of probabilities in section 6). One can only evaluate, and perhaps explain, this feature by considering non-FLRW models. It is noteworthy that many of the "problems" inflation claims to tackle are not problems if the universe simply is always FLRW. Hence, as already argued above, one has a deep problem in explaining why the universe is in the unlikely FLRW state if one accepts the arguments about probabilities current in work on inflation. 
Moreover, suppose we speculated that the real universe is significantly inhomogeneous at the present epoch (at a level beyond that arising from perturbations in FLRW). What would the objections be? There are only two relevant pieces of data, as far as I can see. One is the deep galaxy counts made by the automatic plate measuring machines, which are claimed to restrict variations to a few percent, and the other is the isotropy of the CMWBR. Although the latter is a good test for large lumps in a basically FLRW universe, one has to question (recalling the results of Ellis et al.) whether it really implies homogeneity.

There is a theorem by Ehlers, Geren and Sachs (1968) showing that if a congruence of geodesically-moving observers all observe an isotropic distribution of collisionless gas the metric must be Robertson-Walker. Treciokas and Ellis (1971) have investigated the related problem with collisions. Recently Ferrando et al. (1992) have investigated inhomogeneous models where an isotropic gas distribution is possible. These studies throw into focus a conjecture which is usually assumed, namely that an approximately isotropic gas distribution, at all points, would imply an approximately Robertson-Walker metric. (It is this assumption which underlies some of the arguments used, for example, by Barrow in his talk at this meeting.)

Whether the standard model is correct or not, I feel confident in concluding that one of the more outstanding inhomogeneities is the dedicatee of this piece, Dennis Sciama, and I hope some small part of his talents has been shown here to have been passed on to me. To show how it has influenced the subject, I have marked authors cited in the bibliography below who also appear in the Sciama family tree by an asterisk.

I would like to thank G.F.R. Ellis for comments on the first draft of this survey.

\section{References}

[Anile, 1974] Anile*, A.M. (1974). Anisotropic expansion of the universe and the anisotropy and linear polarization of the cosmic microwave background. Astrophys. Sp. Sci., 29, 415.

[Anninos et al., 1991a] Anninos, P., Matzner, R.A., Rothman, T., and Ryan, M.P. (1991a). How does inflation isotropize the universe? Phys. Rev. D, 43, 3821-3832.

[Anninos et al., 1991b] Anninos, P., Matzner, R.A., Tuluie, R., and Centrella*, J.M. (1991b). Anisotropies of the cosmic background radiation in a "hot" dark matter universe. Astrophys. J., 382, 71-78. 
[Barrow, 1976] Barrow*, J.D. (1976). Light elements and the isotropy of the universe. Mon. Not. R.A.S., 175, 359.

[Barrow, 1982] Barrow*, J.D. (1982). Chaotic behaviour in general relativity. Phys. Repts., 85, 1.

[Barrow, 1984] Barrow*, J.D. (1984). Helium formation in cosmologies with anisotropic curvature. Mon. Not. R.A.S., 211, 221.

[Barrow et al., 1985] Barrow*, J.D., Juszkiewicz, R., and Sonoda*, D.H. (1985). Reply to "the effect of "spottiness" in large-scale structure of the microwave background" by V.N. Lukash and I.D. Novikov. Nature, 316, 48.

[Barrow and Tipler, 1978] Barrow*, J.D and Tipler, F.J (1978). Eternity is unstable. Nature, 278, 453.

[Barrow and Tipler, 1979] Barrow*, J.D and Tipler, F.J. (1979). An analysis of the generic singularity studies by Belinskii, Lifshitz and Khalatnikov. Phys. Repts., 56,371 .

[Belinskii et al., 1980] Belinskii, V.A., Lifshitz, E.M., and Khalatnikov, I.M. (1980). On the problem of the singularities in the general cosmological solution of the Einstein equations. Phys. Lett. A, 77, 214.

[Belinskii, 1991] Belinskii, V.A. (1991). Gravitational breather and topological properties of gravisolitons. Phys. Rev D, 44, 3109-3115.

[Berger, 1991] Berger, B.K. (1991). Comments on the calculation of Liapunov exponents for the Mixmaster universe. Gen. Rel. Grav., 23, 1385.

[Bogoyavlenskii, 1985] Bogoyavlenskii, O.I. (1985). Methods of the qualitative theory of dynamical systems in astrophysics and gas dynamics. Springer-Verlag, Berlin \& Heidelberg. [Russian original published by Nauka, Moscow, 1980.].

[Bona and Coll, 1988] Bona, C. and Coll, B. (1988). On the Stephani universes. Gen. Rel. Grav., 20, 297-303.

[Bonnor, 1972] Bonnor, W.B. (1972). A non-uniform relativistic cosmological model. Mon. Not. R.A.S., 159, 261.

[Bonnor and Chamorro, 1990 and 1991] Bonnor, W.B. and Chamorro, A. (1990). Models of voids in the expanding universe. Ap. J., 361, 21-26.

[Bonnor and Chamorro, 1991] Bonnor, W.B. and Chamorro, A. (1991). Models of voids in the expanding universe II. Astrophys. J., 378, 461-465. 
[Boyd et al., 1991] Boyd, P., Centrella*, J.M., and Klasky, S. (1991). Properties of gravitational "solitons". Phys. Rev. D, 43, 379-390.

[Bradley and Sviestins, 1984] Bradley, J.M. and Sviestins, E. (1984). Some rotating, time-dependent Bianchi VIII cosmologies with heat flow. Gen. Rel. Grav., 16, 1119-1133.

[Burd et al., 1990] Burd*, A.B., Buric, N., and Ellis*, G.F.R. (1990). A numerical analysis of chaotic behaviour in Bianchi IX models. Gen. Rel. Grav., 22, 349-363.

[Carmeli et al., 1983] Carmeli, M., Charach, C. and Feinstein, A. (1983). Inhomogeneous Mixmaster universes: some exact solutions. Ann. Phys. (N.Y.), 150, 392.

[Carr and Hawking, 1974] Carr*, B.J. and Hawking*, S.W. (1974). Black holes in the early universe. Mon. Not. R.A.S., 168, 399.

[Carr and Verdaguer, 1983] Carr*, B.J. and Verdaguer, E. (1983). Soliton solutions and cosmological gravitational waves. Phys. Rev. D, 28, 2995.

[Carr and Yahil, 1990] Carr*, B.J. and Yahil, A. (1990). Self-similar perturbations of a Friedmann universe. Astrophys. J., 360, 330-342.

[Chinea et al., 1991] Chinea, F.J., Fernandez-Jambrina, F., and Senovilla, J.M.M. (1991). A singularity-free spacetime. Madrid/Barcelona preprint FT/UCM/16/91.

[Clarke, 1976] Clarke*, C.J.S. (1976). Space-time singularities. Comm. math. phys., 49, 17.

[Clarke et al., 1990] Clarke*, C.J.S., Ellis*, G.F.R., and Vickers*, J. (1990). The large-scale bending of cosmic strings. Class. Quant. Grav., 7, 1-14.

[Coley and Tavakol, 1992] Coley*, A.A. and Tavakol, R.K. (1992). Fragility in cosmology. QMW preprint.

[Collins, 1971] Collins*, C.B. (1971). More qualitative cosmology. Comm. math. phys., 23, 137.

[Collins and Hawking, 1972] Collins*, C.B. and Hawking*, S.W. (1972). The rotation and distortion of the universe Mon. Not. R.A.S., 162, 307.

[Collins and Hawking, 1973] Collins*, C.B. and Hawking*, S.W. (1973). Why is the universe isotropic? Astrophys. J., 180, 37.

[Collins and Stewart, 1971] Collins*, C.B. and Stewart*, J.M. (1971). Qualitative cosmology. Mon. Not. R.A.S., 153, 419-434. 
[Dunsby, 1992] Dunsby*, P.K.S. (1992). Perturbations in general relativity and cosmology. Ph.D. thesis, Queen Mary and Westfield College, University of London.

[Dyer, 1976] Dyer, C.C. (1976). The gravitational perturbation of the cosmic background radiation by density concentrations. Mon. Not. R.A.S., 175, 429.

[Eardley et al., 1971] Eardley, D.M., Liang, E., and Sachs, R.K. (1971). Velocitydominated singularities in irrotational dust cosmologies. J. Math. Phys., 13, 99.

[Ehlers et al., 1968] Ehlers, J., Geren, P., and Sachs, R.K. (1968). Isotropic solutions of the Einstein-Liouville equation. J. Math. Phys., 9, 1344.

[Ellis, 1967] Ellis*, G.F.R. (1967). Dynamics of pressure-free matter in general relativity. J. Math. Phys., 8, 1171.

[Ellis, 1991] Ellis*, G.F.R. (1991). Standard and inflationary cosmologies. In Mann, R. and Wesson, P., editors, Gravitation: a Banff summer institute. World Scientific, Singapore.

[Ellis et al., 1978] Ellis*, G.F.R., Maartens*, R.A., and Nel*, S.D. (1978). The expansion of the universe. Mon. Not. R.A.S., 184, 439.

[Feinstein, 1988] Feinstein, A. (1988). Late-time behaviour of primordial gravitational waves in expanding universe. Gen. Rel. Grav., 20, 183-190.

[Ferrando et al., 1992] Ferrando, J., Morales, J., and Portilla, M. (1992). Inhomogeneous space-times admitting isotropic radiation. Valencia preprint.

[Ferrari, 1990] Ferrari, V. (1990). Colliding waves in general relativity. In N. Ashby, D. Bartlett and Wyss, W., editors, General Relativity and Gravitation, 1989, pp. 3-20. Cambridge University Press, Cambridge, New York and Melbourne.

[Futamase et al., 1989] Futamase, T., Rothman, T., and Matzner, R. (1989). Behaviour of chaotic inflation in anisotropic cosmologies with nonminimal coupling. Phys. Rev. D, 39, 405-411.

[Goetz, 1990] Goetz, G. (1990). Gravitational field of plane symmetric thick domain walls. J. Math. Phys., 31, 2683-2687.

[Goode, 1983] Goode, S.W. (1983). Spatially inhomogeneous cosmologies and their relation with the Friedmann-Robertson-Walker models. Ph.D. thesis, University of Waterloo.

[Goode et al., 1992] Goode, S.W., Coley*, A.A., and Wainwright, J. (1992). The isotropic singularity in cosmology. Class. Quant. Grav., 9, 445-455. 
[Goode and Wainwright, 1982] Goode, S. and Wainwright, J. (1982). Singularities and evolution of the Szekeres cosmological models. Phys. Rev. D, 26, 3315.

[Gott, 1985] Gott, J.R. (1985). Gravitational lensing effects of vacuum: exact solutions. Astrophys. J., 288, 422.

[Griffiths, 1991] Griffiths, J.B. (1991). Colliding plane waves in general relativity, Oxford mathematical monographs. Oxford University Press, Oxford.

[Harvey, 1979] Harvey, A.L. (1979). Automorphisms of the Bianchi model Lie groups. J. Math. Phys., 20, 251.

[Hausman et al., 1983] Hausman, M., Olson, D.W., and Roth, B. (1983). The evolution of voids in the expanding universe. Astrophys. J., 270, 351.

[Hawking and Ellis, 1973] Hawking*, S.W. and Ellis*, G.F.R. (1973). The large-scale structure of space-time. Cambridge University Press, Cambridge.

[Hawking and Tayler, 1966] Hawking*, S.W. and Tayler, R.J. (1966). Helium production in an anisotropic big-bang cosmology. Nature, 209, 1278.

[Hewitt et al., 1991] Hewitt, C., Wainwright, J., and Glaum, M. (1991). Qualitative analysis of a class of inhomogeneous self-similar cosmological models II. Class. Quant. Grav., 8, 1505-1518.

[Hiscock, 1985] Hiscock, W.A. (1985). Exact gravitational field of a string. Phys. Rev. D, 31, 3288-90.

[Holmes et al., 1990] Holmes, G., Joly*, G.J., and Smallwood, J. (1990). On the application of computer algebra to velocity dominated approximations. Gen. Rel. Grav., 22, 749-764.

[Hoyle, 1962] Hoyle, F. (1962). Cosmological tests of gravitational theories. In Evidence for gravitational theories, ed. C. Moller, Enrico Fermi Corso XX, Varenna, p.141. Academic Press, New York.

[Ibanez and Verdaguer, 1983] Ibanez, J. and Verdaguer, E. (1983). Soliton collision in general relativity. Phys. Rev. Lett., 51, 1313.

[Ipser and Sikivie, 1984] Ipser, J. and Sikivie, P. (1984). Gravitationally repulsive domain walls. Phys. Rev. D, 30, 712-9.

[Jaklitsch, 1987] Jaklitsch*, M. (1987). First order field equations for Bianchi types $I I-V I_{h}$. Capetown preprint 87-6. 
[Jantzen, 1979] Jantzen, R. (1979). The dynamical degrees of freedom in spatially homogeneous cosmology. Comm. math. phys., 64, 211.

[Jantzen, 1984] Jantzen, R. (1984). Spatially homogeneous dynamics: a unified picture. In Ruffini, R. and Fang L.-Z., editors, Cosmology of the early universe, pp. 233-305. Also in "Gamow cosmology", (Proceedings of the International School of Physics 'Enrico Fermi', Course LXXXVI) ed. R. Ruffini and F. Melchiorri, pp. 61-147, North Holland, Amsterdam, 1987.

[Juszkiewicz et al., 1983] Juszkiewicz, R., Bajtlik, S., and Gorski, K. (1983). The helium abundance and the isotropy of the universe. Mon. Not. R.A.S., 204, 63P.

[Kantowski, 1969a] Kantowski, R. (1969a). The Coma cluster as a spherical inhomogeneity in relativistic dust. Astrophys. J., 155, 1023.

[Kantowski, 1969b] Kantowski, R. (1969b). Corrections in the luminosity-redshift relations of the homogeneous Friedman models. Astrophys. J., 155, 59.

[King and Ellis, 1973] King*, A.R. and Ellis*, G.F.R. (1973). Tilted homogeneous cosmological models. Comm. math. phys., 31, 209.

[Kitchingham, 1984] Kitchingham*, D.W. (1984). The use of generating techniques for space-times with two non-null commuting Killing vectors in vacuum and stiff perfect fluid cosmological models. Class. Quant. Grav., 1, 677-694.

[Krasinski, 1990] Krasinski, A. (1990). Early inhomogeneous cosmological models in Einstein's theory. In Bertotti, B., Bergia, S., Balbinot, R., and Messina, A., editors, Modern cosmology in retrospect. Cambridge University Press, Cambridge.

[Lemaître, 1933] Lemaître, G. (1933). L'univers en expansion. Ann. Soc. Sci. Bruxelles $A, 53,51$.

[Lifshitz and Khalatnikov, 1963] Lifshitz, E.M. and Khalatnikov, I.M. (1963). Investigations in relativistic cosmology. Adv. Phys., 12, 185.

[Lin and Wald, 1991] Lin, X.-F. and Wald, R.M. (1991). Proof of the closed universe recollapse conjecture for general Bianchi IX cosmologies. Phys. Rev. D, 41, 2444.

[Linet, 1985] Linet, B. (1985). The static metrics with cylindrical symmetry describing a model of cosmic strings. Gen. Rel. Grav., 17, 1109.

[Lynden-Bell et al., 1988] Lynden-Bell, D., Faber, S., Burstein, D., Davies, R., Dressler, A., Terlevich, R., and Wegener, G. (1988). Spectrosopy and photometry of elliptical galaxies V. Galaxy streaming toward the new supergalactic center. Astrophys. J., 326, 19. 
[MacCallum, 1971] MacCallum*, M.A.H. (1971). A class of homogeneous cosmological models III: asymptotic behaviour. Comm. math. phys., 20, 57-84.

[MacCallum, 1979] MacCallum*, M.A.H. (1979). Anisotropic and inhomogeneous relativistic cosmologies. In Hawking, S.W. and Israel, W., editors, General relativity: an Einstein centenary survey, pp. 533-580. Cambridge University Press, Cambridge.

[MacCallum, 1982] MacCallum*, M.A.H. (1982). Relativistic cosmology for astrophysicists. In de Sabbata, V., editor, Origin and evolution of the galaxies, pp. 9-33. World Scientific, Singapore. Also, in revised form, in "Origin and evolution of the galaxies", ed. B.J.T. and J.E. Jones*, Nato Advanced Study Institute Series, B97, pp. 9-39, D.Reidel and Co., Dordrecht, 1983.

[MacCallum, 1984] MacCallum*, M.A.H. (1984). Exact solutions in cosmology. In Hoenselaers, C. and Dietz, W., editors, Solutions of Einstein's equations: techniques and results (Retzbach, Germany, 1983), volume 205 of Lecture Notes in Physics, pp. 334-366. Springer Verlag, Berlin and Heidelberg.

[MacCallum, 1987] MacCallum*, M.A.H. (1987). Strengths and weaknesses of cosmological big-bang theory. In W.R. Stoeger*, S.J., editor, Theory and observational limits in cosmology, pp. 121-142. Specola Vaticana, Vatican City.

[Maddox et al., 1990] Maddox, S., Efstathiou*, G., Sutherland, W., and Loveday, J. (1990). Galaxy correlations on large scales. Mon. Not. R.A.S., 242, 43P.

[Matravers and Madsen, 1985] Matravers, D.T. and Madsen*, M.S. (1985). Baryon number generation in a class of anisotropic cosmologies. Phys. Lett. B, 155, 43-46.

[Matravers et al., 1985] Matravers, D.T., Madsen*, M.S., and Vogel, D.L (1985). The microwave background and $(m, z)$ relations in a tilted cosmological model. Astrophys. Sp. Sci., 112, 193-202.

[Matravers et al., 1984] Matravers, D.T., Vogel, D.L., and Madsen*, M.S. (1984). Helium formation in a Bianchi V universe with tilt. Class. Quant. Grav., 1, 407.

[Matzner, 1991] Matzner, R.A (1991). Three-dimensional numerical cosmology. Ann. N.Y. Acad. Sci., 631, 1-14.

[Mavrides, 1977] Mavrides, S. (1977). Anomalous Hubble expansion and inhomogeneous cosmological models. Mon. Not. R.A.S., 177, 709.

[Meszaros, 1991] Meszaros, A. (1991). On shell crossing in the Tolman metric. Mon. Not. R. Astr. Soc., 253, 619-624. 
[Moss and Sahni, 1986] Moss*, I. and Sahni, V. (1986). Anisotropy in the chaotic inflationary universe. Phys. Lett. B, 178, 159.

[Newman, 1979] Newman, R.P.A.C. (1979). Singular perturbations of the empty Robertson-Walker cosmologies. Ph. D. thesis, University of Kent.

[Novikov, 1968] Novikov, I. (1968). An expected anisotropy of the cosmological radioradiation in homogeneous anisotropic models. Astr. Zh., 45, 538. Translation in Sov. Astr.-A.J. 12, 427.

[Obukhov, 1992] Obukhov, Y. (1992). Rotation in cosmology. Gen. Rel. Grav., 24, $121-128$.

[Olson, 1977] Olson, D.W. (1977). Helium production and limits on the anisotropy of the universe Astrophys. J., 219, 777.

[Penrose, 1979] Penrose, R. (1979). Singularities and time-asymmetry. In Hawking, S.W. and Israel, W., editors, General relativity: an Einstein centenary survey, pp. 581-638. Cambridge University Press, Cambridge.

[Perko et al., 1972] Perko, T., Matzner, R.A., and Shepley, L.C. (1972). Galaxy formation in anisotropic cosmologies. Phys. Rev. D, 6, 969.

[Rasband, 1971] Rasband, S.N. (1971). Expansion anisotropy and the spectrum of the cosmic background radiation. Astrophys. J., 170, 1.

[Rees, 1968] Rees*, M.J. (1968). Polarization and spectrum of the primeval radiation in an ansiotropic universe. Astrophys. J., 153, 1.

[Ribeiro, 1992a] Ribeiro*, M.B. (1992a). On modelling a relativistic hierarchical (fractal) cosmology by Tolman's spacetime. I. Theory. Astrophys. J., 388, 1.

[Ribeiro, 1992b] Ribeiro*, M.B. (1992b). On modelling a relativistic hierarchical (fractal) cosmology by Tolman's spacetime. II. Analysis of the Einstein-De Sitter model. Astrophys. J., (to appear).

[Romano and Pavon, 1992] Romano, V. and Pavon, D. (1992). Causal dissipative Bianchi cosmology. Catania/Barcelona preprint.

[Roque and Ellis, 1985] Roque*, W.L. and Ellis*, G.F.R. (1985). The automorphism group and field equations for Bianchi universes. In MacCallum, M., editor, Galaxies, axisymmetric systems and relativity: essays presented to W.B. Bonnor on his 65th birthday, pp. 54-73. Cambridge University Press, Cambridge. 
[Rosquist et al., 1990] Rosquist, K., Uggla, C., and Jantzen, R. (1990). Extended dynamics and symmetries in perfect fluid Bianchi cosmologies. Class. Quant. Grav., 7, 625-637.

[Rothman and Ellis, 1986] Rothman, T. and Ellis*, G.F.R. (1986). Does inflation occur in anisotropic cosmologies? Phys. Lett. B, 180, 19.

[Rothman and Matzner, 1982] Rothman, T. and Matzner, R.A. (1982). Effects of anisotropy and dissipation on the primordial light isotope abundances. Phys. Rev. Lett., 48, 1565.

[Ryan and Shepley, 1975] Ryan, M.P. and Shepley, L.C. (1975). Homogeneous relativistic cosmologies. Princeton University Press, Princeton.

[Saez and Arnau, 1990] Saez, D. and Arnau, J. (1990). On the Tolman Bondi solution of Einstein equations. Numerical applications. In E. Verdaguer, Garriga, J. and Cespedes, J., editors, Recent developments in gravitation (Proceedings of the "Relativity Meeting - 89"), pp. 415-422. World Scientific, Singapore.

[Salam, 1990] Salam, A. (1990). Unification of fundamental forces. Cambridge University Press, Cambridge.

[Salvati et al., 1987] Salvati, G., Schelling, E., and van Leeuwen, W. (1987). Homogeneous viscous universes with magnetic field. II Bianchi type I spaces. Ann. Phys. (N.Y.), 179, 52-75.

[Sato, 1984] Sato, H. (1984). Voids in the expanding universe. In General relativity and gravitation: Proceedings of the 10th international conference on general relativity and gravitation ed. B. Bertotti, F. de Felice and A. Pascolini, pp. 289-312, D. Reidel and Co., Dordrecht.

[Saunders et al., 1991] Saunders, W., Frenk, C., Rowan-Robinson, M., Efstathiou*, G., Lawrence, A., Kaiser*, N., Ellis, R., Crawford, J., and Parry, I. (1991). The density field of the local universe. Nature, $\mathbf{3 4 9}, 32$.

[Siklos, 1978] Siklos*, S.T.C. (1978). Occurrence of whimper singularities. Comm. math. phys., 58, 255.

[Siklos, 1980] Siklos*, S.T.C. (1980). Field equations for spatially homogeneous spacetimes. Phys. Lett. A, 76, 19.

[Sirousse-Zia, 1982] Sirousse-Zia, H. (1982). Fluctuations produced by the cosmological constant in the empty Bianchi IX universe. Gen. Rel. Grav., 14, 751. 
[Stewart and Ellis, 1968] Stewart*, J.M. and Ellis*, G.F.R. (1968). On solutions of Einstein's equations for a fluid which exhibit local rotational symmetry. J. Math. Phys., 9, 1072.

[Sussman, 1985] Sussman*, R.A. (1985). Conformal structure of a Schwarzschild black hole immersed in a Friedman universe. Gen. Rel. Grav., 17, 251-292.

[Thorne, 1967] Thorne, K.S. (1967). Primordial element formation, primordial magnetic fields and the isotropy of the universe. Astrophys. J., 48, 51.

[Tod, 1992] Tod*, K.P. (1992). Mach's principle and isotropic singularities. In this volume.

[Tolman and Matzner, 1984] Tolman, B. and Matzner, R.A. (1984). Large scale anisotropies and polarization of the microwave background in homogeneous cosmologies. Proc. Roy. Soc. A, 392, 391.

[Tolman, 1934] Tolman, R.A. (1934). Effect of inhomogeneity on cosmological models. Proc. Nat. Acad. Sci. (Wash.), 20, 169.

[Treciokas and Ellis, 1971] Treciokas*, R. and Ellis*, G.F.R. (1971). Isotropic solutions of the Einstein-Boltzmann equations. Comm. math. phys., 23, 1.

[Turner, 1992] Turner, M. (1992). The tilted universe. Gen. Rel. Grav., 24, 1-7.

[Vilenkin, 1983] Vilenkin, A. (1983). Gravitational field of vacuum domain walls. Phys. Lett. B, 133, 177-179.

[Wainwright and Anderson, 1984] Wainwright, J. and Anderson, P. (1984). Isotropic singularities and isotropization in a class of Bianchi $V I_{h}$ cosmologies. Gen. Rel. Grav., 16, 609-24.

[Wainwright and Hsu, 1989] Wainwright, J. and Hsu, L. (1989). A dynamical systems approach to Bianchi cosmologies: orthogonal models of class A. Class. Quant. Grav., 6, 1409-1431.

[Wald, 1983] Wald, R.M. (1983). Asymptotic behaviour of homogeneous cosmological models in the presence of a positive cosmological constant. Phys. Rev. D, 28, 211.

[Wang, 1991] Wang, A.-Z. (1991). Planar domain walls emitting and absorbing electromagnetic radiation. Ioannina preprint IOA-258/91.

[Wesson, 1978] Wesson*, P. (1978). General relativistic hierarchical cosmology: an exact model. Astrophys. Sp. Sci., 54, 489.

[Wesson, 1979] Wesson*, P. (1979). Observable relations in an inhomogeneous selfsimilar cosmology. Astrophys. J., 228, 647. 